\title{
Naissance des éoliennes : question de définition(s)
}

\section{The birth of wind turbines: a question of definition(s)}

\author{
Philippe Bruyerre ${ }^{1}$ \\ ${ }^{1}$ Docteur en histoire des techniques, Centre Alexandre Koyré, pbruyerre@yahoo.fr
}

RESUME. Cet article questionne les récits historiques concernant la naissance de différentes éoliennes qu'il s'agisse des moulins de drainage aux Pays-Bas (XVIle siècle), des éoliennes américaines de pompage (XIXe siècle) et des éoliennes électriques actuelles au Danemark (XXe siècle). Ces multiples naissances et renaissances renvoient à la définition de l'objet technique, au double sens du mot définition : à la fois énonciation de ce qu'est une chose et degré de finesse spatiale et temporelle (comme dans « haute définition » dans le domaine de l'image).

ABSTRACT. This article investigates the historical accounts of the births of different wind turbines: the Dutch polder windmill (17th century), the American wind pump (19th century) and the Danish wind turbine (20th century). These multiple births and rebirths refer to the "definition" of the technical object, in both senses of the word "definition": the enunciation of what a thing is and its degree of spatial and temporal finesse (as in "high definition" in the domain of images).

MOTS-CLÉS. Vent, énergie, histoire, définition.

KEYWORDS. Wind, energy, history, definition.

\section{Introduction}

Le moulin à vent est hollandais, l'éolienne de pompage est américaine, l'éolienne moderne est danoise. Ces définitions nationales peuvent être considérées comme de simples facilités de langage pour spécialistes, autant d' «innovations » qu'il faut bien identifier, classifier et inscrire dans une histoire technique. Elles sont aussi des constructions d'identité nationale, des objets emblématiques dans lesquels la Nation se reconnaît et par lesquels elle se définit.

Pour interroger ces multiples définitions, cet article utilise comme source la philatélie en prenant au pied de la lettre l'affirmation d'Ernst Rutherford pour qui All science is either physics or stamp collecting ( Toute science est soit de la physique, soit de la philatélie »). Le timbre est une image banale véhiculant de manière silencieuse une histoire partagée, inventée par une nation pour se représenter, outil de politique interne et d'image de marque externe, gouvernée par décret ministériel [ARR 18]. Le timbre qui frappe par son image est aussi une ressource historique, donnant dans certains cas une définition très précise de l'objet technique présenté et dans d'autres cas n'évoquant l'objet que dans ses grandes lignes.

\section{Le moulin de drainage hollandais}

\subsection{Moulin de drainage, de la machine au monument}

En juin 2013, à l'occasion du $90^{\text {ème }}$ anniversaire de l'Association hollandaise de conservation des moulins (De Hollandsche Molen), la Poste néerlandaise, PostNL, émet une série de 10 timbres sur les moulins néerlandais (Nederlandse Molens) en deux séries de cinq timbres [POS 13], la première sur les moulins installés au bord de l'eau, la seconde sur ceux installés dans la campagne ou en ville. 

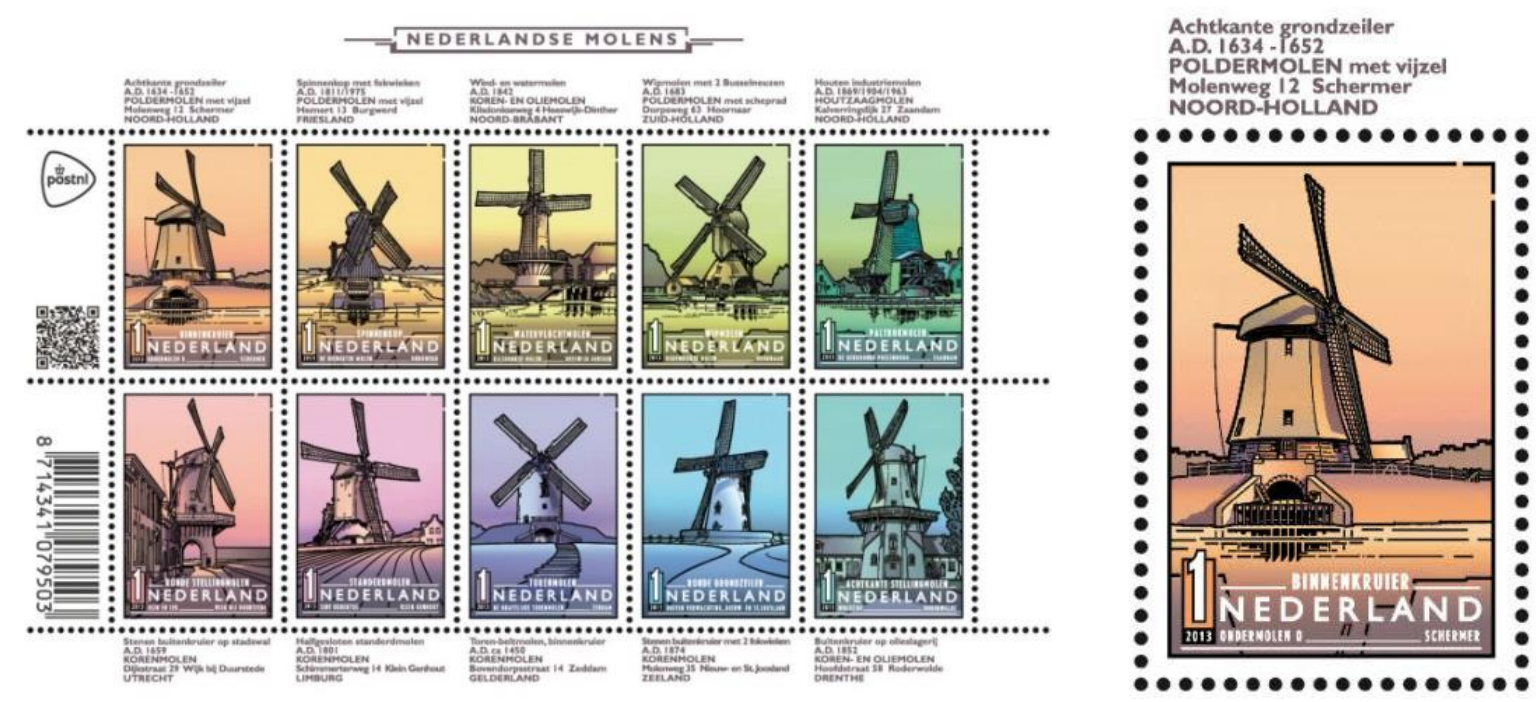

Figure 1. Moulins néerlandais, 2013. Série complète à gauche, Ondermolen D à droite.

Chaque timbre est accompagné d'une description détaillée. Par exemple, sur le premier de ces timbres (agrandi sur la figure 1), il est indiqué dans la partie supérieure qu'il s'agit d'un moulin octogonal (Achtkante) à ailes rasant le sol (grondzeiler) construit vers 1634-1652, un moulin de polder à vis d'Archimède (POLDERMOLEN met vijzel) situé à Schermer dans la province de Noord-Holland. Sur le timbre lui-même, au-dessus du mot NEDERLAND, il est précisé qu'il s'agit d'un moulin à calotte tournante à système d'orientation intérieur (Binnenkruier) et tout en bas à droite figure le nom de ce moulin Ondermolen $D$, «moulin inférieur $\mathrm{D} »$ et celui du polder, Schermer. Ces définitions structurelle et fonctionnelle sont fournies par De Hollandsche Molen, qui a construit depuis 2001 une base de données des moulins hollandais et belges [DEH 01]. Les moulins y sont classés suivant quatorze types (Grondzeiler pour l'Ondermeulen D), trente et une fonctions (Poldermolen) et trente-quatre caractéristiques (Achkante binnenkruier): à chaque moulin ses «marques de connaissance » (kenmerken), autant d'innovations différentiant entre elles ces machines toutes mues par le vent.

Longtemps présenté comme d'origine hollandaise, mentionné en 1408 à Alkmaar, le moulin de drainage est en fait flamand, attesté en 1316 selon un article récent [DEN 06] au titre quelque peu national : Niet Nederland maar vlaanderen de bakermat! Le berceau [du poldermolen] ne se trouve pas aux Pays-Bas, mais en Flandres ! Ce qui rend le moulin hollandais est sa structure en bois à huit côtés facilement reconnaissable : il s'agit d'un moulin tour dont la tête repose sur des galets en bois et s'oriente manuellement face au vent. L'arbre principal entraîne au travers de deux renvois d'angle (rouet-lanterne) un arbre secondaire horizontal, sur lequel est fixée la roue à palettes, sorte de roue à aubes. Cette roue partiellement immergée prend l'eau et la rejette dans un canal qui forme réservoir (boezem).

Le moulin de drainage fait l'objet de nombreuses propositions d'améliorations. Entre 1560 et 1700 , quelques 102 brevets sont attribués par les Etats Généraux des Pays-Bas pour des moulins de drainage, sans compter les nombreux brevets de pompes [HIL 96]. Malgré cette offre d'innovations importante, le moulin de drainage reste dans sa configuration initiale jusqu'en 1852, date à laquelle une vis d'Archimède, un brevet de Symon Hulsbos de 1634, remplace la roue à palettes, puis en 1877 avec l'installation d'arbres moteur en fonte. Ces moulins résistent à l'avancée de la machine à vapeur, mais sont détrônés en 1928 par des pompes électriques.

Au milieu des années 1960, seuls 300 moulins sont encore en état de fonctionnement aux Pays-Bas, et il n'existe plus aucun meunier professionnel [MUL 91]. Suite à l'adoption de la loi sur les monuments (Monumentenwet) en 1961, le Rijksdienst voor de Monumentenzorg (RDMZ), l'administration en charge du patrimoine, écrit dans un rapport de 1966 qu'un moulin qui ne peut être remis en route ne peut plus être protégé comme monument : ce n'est donc pas sa valeur patrimoniale qui compte, mais la 
possibilité de lui faire remplir de nouveau sa fonction originelle. Le moulin Ondermolen $D$ devient un hybride de machine et de monument puisqu'il doit fonctionner pour avoir le statut de monument ; il est inscrit en 1967 sur le Registre royal des monuments et un musée, le Museummolen, est créé sur le site en 1968.

En 2012, le Rijksdienst voor het Cultureel Erfgoed (RCE) qui succède au RDMZ révise cette vision sur la conservation des moulins dans le cadre de la politique de «modernisation de la protection des monuments » (Modernisering Monumentenzorg) [NIE 12]. La position est claire : le fonctionnement des moulins ne fournit pas les recettes nécessaires à leur conservation et il faut désormais mettre plus l'accent sur les moulins comme monuments plutôt que comme machines. Avec cette nouvelle définition, les moulins deviennent des monuments de l'histoire de la technologie et leur conservation doit se fonder sur leur valeur patrimoniale en tant que monuments, leur place dans le paysage, et leur fonction dans la société. Le rapport explique que les moulins et leurs conservateurs ne forment plus une entité unique : les moulins sont devenus d'abord des monuments, et les meuniers sont devenus des gestionnaires et des hôtes.

\subsection{Moulin de drainage, icone néerlandaise}

En 2014, PostNL lance deux séries de timbres sur les « icones néerlandaises » (nederlandse iconen) [POS 14], une série nationale de dix timbres et une série internationale de cinq timbres : le moulin à vent fait partie de ces deux séries, comme la bicyclette, la vache (Holstein-Frise), une façade avec pignon à échelons d'Amsterdam et... la tulipe évidemment. Dans le communiqué de presse en anglais de PostNL, le moulin est décrit comme un Smockmill, terme anglais pour un moulin octogonal (achtkante molen). En regardant de plus près, à plus haute définition, et en prenant la version néerlandaise du même communiqué de presse, il s'agit en fait d'un wipmolen, moulin à pivot creux, lui aussi utilisé pour le drainage.
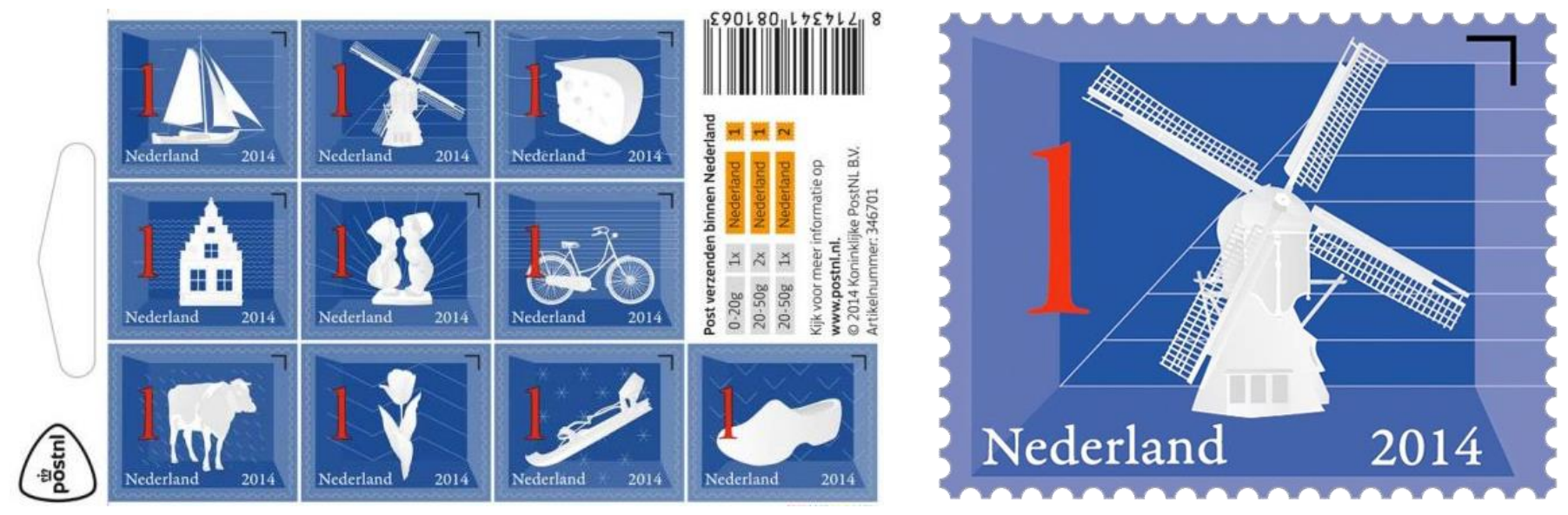

Figure 2. Icones néerlandaises, 2014. Série complète à gauche, moulin à vent à droite.

Moulin tour en anglais, moulin pivot en néerlandais... la définition est floue, mais ce n'est pas ce qui compte. Les concepteurs de ces timbres expliquent s'être concentrés sur la forme en laissant de côté « les couleurs et autres fioritures »_[POS 14] et ajoutent que les zones de couleur bleue créent « une sorte de vitrine pour intégrer les objets dans lesquels les icônes sont affichées, comme dans un musée. » Le concepteur a voulu en outre «montrer chaque icône dans son environnement » en dessinant en arrière-plan du moulin une série de lignes parallèles, image épurée du réseau de canaux du polder.

Ce timbre propose une vue élargie à faible résolution dans laquelle le moulin est réduit à une silhouette sur un fond géométrique. Il pousse à considérer son environnement, celui du polder dont l'origine remonte au VIIIème siècle quand des ilots de tourbe sont exploités en drainant les terres humides. Au fur et à mesure, la couche supérieure comprime la couche inférieure et s'oxyde au contact 
de l'air, entraînant une baisse du niveau du sol d'un mètre par siècle : l'eau s'accumule et forme des lacs. Cette «crise écologique », désignée par la «Phase des Lacs» de 1350 à 1550 [DAM 01], est due en partie à des causes naturelles (transgression marine, tempêtes), mais aussi et surtout à cette collecte industrielle de tourbe. Les Hollandais construisent dès le XIVème siècle des digues, des écluses et des canaux pour se protéger des assauts de la mer, mais plusieurs de ces digues se rompent à la Toussaint de 1570 inondant tout le pays. Cette inondation de la Toussaint (Allerheiligenvloed) marque une « coupure fondamentale dans l'esprit populaire néerlandais » [CIR 91] et un tournant dans la lutte contre la mer en passant de la protection par des digues à la conquête des terres inondées par des moulins à vent, la «poldérisation ».

En 1627, des notables d'Alkmaar, à $40 \mathrm{~km}$ au Nord d'Amsterdam, associés à neuf investisseurs demandent une autorisation (ooctroi) aux Etats de Hollande pour l'assèchement du lac Schermeer, un investissement potentiellement rentable si l'on en juge par les $17 \%$ de rentabilité atteintes pour le polder voisin de Beemster créé en 1612. Les moulins sont installés sur quatre niveaux, chaque ensemble relevant l'eau d'un mètre, une limite imposée par la roue à palettes. Certains moulins sont installés au fonds du polder, une innovation unique aux Pays-Bas, et envoient l'eau vers les Ondermolens, les moulins «inférieurs», puis vers les moulins «moyens» et enfin les moulins «supérieurs ». Le démarrage de l'assèchement en 1634 entraîne des protestations de riverains et du Conseil de l'eau (Uitwaterende Sluizen) faute de bonne gestion des eaux drainées, mais cela n'arrête pas les «messieurs du Schermer». Après différents réglages, le projet est terminé en 1666. Le Schermerpolder, d'une superficie de 4726 hectares, est ensuite divisé en parcelles et loué aux fermiers et éleveurs et peut-être à de riches marchands qui y construisent leur villa de campagne comme sur le Beemsterpolder tout proche.

Le décret de 1915 limitant la durée du travail des meuniers, le Conseil des polders décide en 1924 de passer au pompage électrique avec trois pompes dénommée Emma, Juliana et Wilhelmina suivant la tradition, mises en service en 1928. Les derniers moulins s'arrêtent en 1929. Certains sont vendus, d'autres réquisitionnés au cours de la Seconde guerre mondiale, puis deviennent des moulins «BWO», du nom de la loi sur la protection de l'eau en temps de guerre (Bescherming Waterstaatswerken in Oorlogstijd) lors de la Guerre Froide dans les années 1950. Deux autres moulins sont conservés pour faire face à la pénurie de logement. Au final, il reste aujourd'hui 17 moulins sur le polder du Schermer, des icônes (iconen), comme le dit le Museummolen, dont huit peuvent encore pomper 60000 litres d'eau par minute et font l'objet d'un suivi technique précis : on sait par exemple que l'Ondermolen $D$, de 24,55 mètres de diamètre, a effectué quelques 693368 tours au cours de l'année $2017 \ldots$

\subsection{Moulin de drainage, polder et identité nationale}

Ce que célèbrent les timbres présentés plus haut, c'est la constitution d'une identité néerlandaise, exprimée par le dicton God schiep de aarde, maar de Nederlanders schiepen Nederland («Dieu a créé le Monde et les Néerlandais ont créé les Pays-Bas »). Le «lion Batave», par son ingéniosité et son courage se bat contre le «loup d'eau », le monstre de la mer, un combat célébré par le poète Joost van der Wondel vers 1640 [STE 30]. Le « loup d'eau » (waterwolf) est un terme courant dans l'histoire, la littérature et le folklore des Pays-Bas pour décrire l'action destructrice et dévastatrice de l'eau entrainée par le vent sur le littoral [TEB 02]. S'ajoute à ces représentations la notion de polder model, modèle sociétal néerlandais selon lequel les membres de la société sont contraints de négocier entre eux pour prendre des décisions communes comme ce qui se passe au sein d'un polder.

L'histoire du Schmerpolder montre qu'en fait de combat contre le loup d'eau, c'est un combat contre l'homme lui-même que mènent les moulins. Après avoir créé des lacs en exploitant la tourbe pendant des siècles, les néerlandais vident les lacs grâce à l'innovation des moulins à vent, mais la tourbe est toujours là et les polders s'enfoncent... Aujourd'hui, vingt-six pour cent du pays se trouve en dessous du niveau de la mer avec le point le plus à bas à - 6,76 mètres (Zuidplaspolder au Nord-Est de 
Rotterdam), le record d'Europe [NET 13]. A l'heure du changement climatique, l'innovation consisterait plutôt à « dépoldériser » (la tourbe en contact avec l'air produit du $\mathrm{CO}_{2}$ ), mais les expériences menées durant les années 2000 font l'objet de résistances sociales [GOE 10] compte tenu du statut iconique du polder et des actions dites de managed realignment (rechargement des dunes, création de nouvelles digues...) semblent leur être préférées.

\section{L'éolienne américaine de pompage}

\subsection{Une invention américaine}

En 1981, la poste américaine, United States Postal Office, émet une série de cinq timbres sur le thème des moulins [USP 81]. Les quatre premiers timbres évoquent des moulins à vent traditionnels construits en Nouvelle-Angleterre au XVIIIe siècle, ainsi qu'un moulin hollandais de l'Illinois de 1860 (moulin Faybian). Le dernier timbre représente une éolienne du sud-ouest (Southwestern windmill), au Texas en 1890, une éolienne de pompage derrière un réservoir d'eau surélevé.

En 2008, la série «Drapeaux de notre nation » associe à chaque Etat une image symbolisant un paysage ou une activité associé à cet Etat : pour l'Illinois, le choix se porte sur l'éolienne de pompage, Batavia, près de Chicago, se revendiquant comme la «cité des moulins à vent » [PET 13]. Quelques années plus tard, en 2011, un timbre célébrant le cent-cinquantième anniversaire de la création de l'Etat du Kansas symbolise, selon l'US Postal Office [USP 10], les caractéristiques les plus importantes de cet Etat : « histoire, industrie, agriculture et ingéniosité pionnière ». En arrière-plan de l'éolienne de pompage, cinq éoliennes modernes témoignent de la continuité et de l'avenir de l'économie du Kansas.

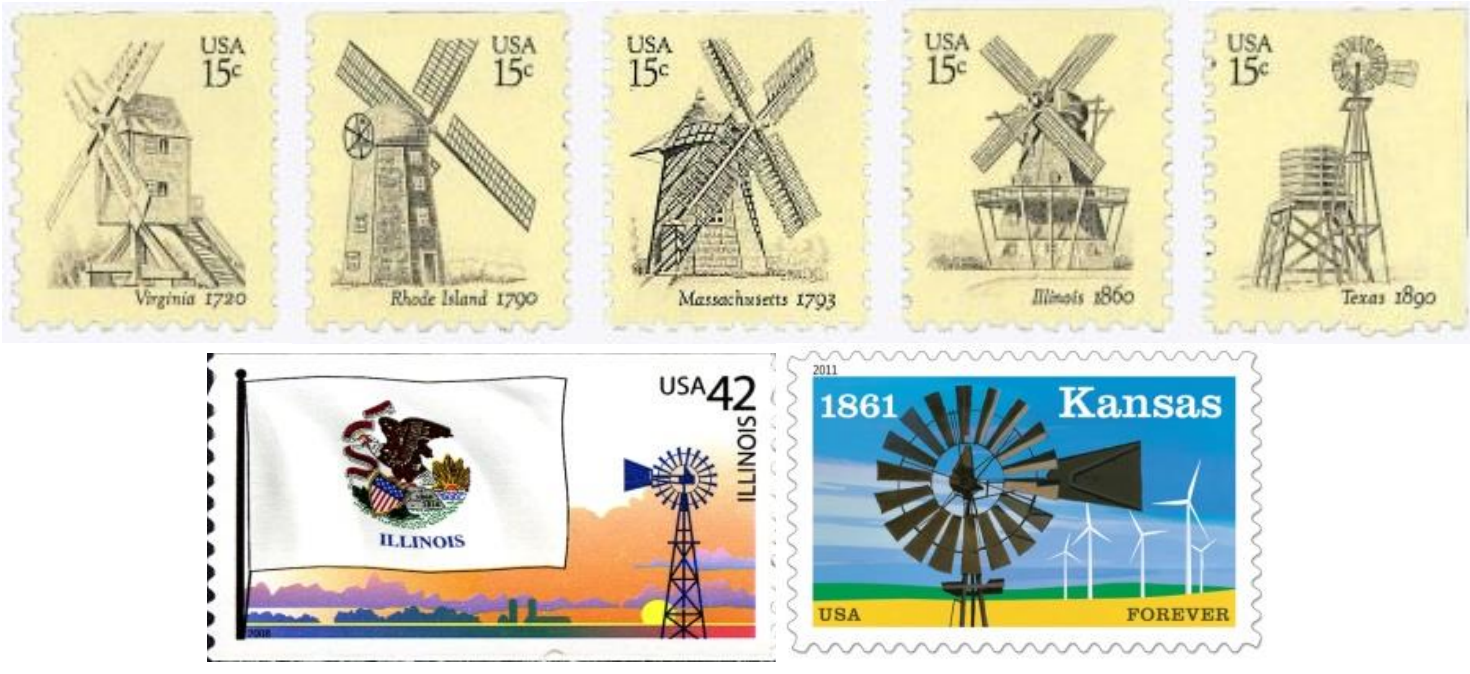

Figure 3. Timbres américains de 1981, 2008 et 2011.

Illinois, Texas, Kansas : trois Etats américains associés à l'éolienne de pompage, le premier du point de vue industriel, les deux seconds du point de vue de l'usage.

Le brevet de Daniel Halladay de 1854 [HAL 54] est considéré par tous les historiens comme celui de l'éolienne de pompage. Daniel Halladay, né dans le Vermont en 1826, est un mécanicien qualifié ayant travaillé notamment sur la machine calorique d'Ericsson qu'il installe à l'Exposition universelle de Londres en 1851. C'est à la demande de John Burham, un spécialiste des pompes [BAK 80], qu'il développe cette technique et dépose un brevet sur un "régulateur amélioré pour moulin à vent» (Improved Governor for Windmill). A examiner de près le brevet de 1854, il s'agit d'un moulin à quatre pales qui peuvent pivoter autour de leur axe longitudinal, la commande étant effectuée par des biellettes fixées sur plateau central. L'innovation est double : assurer le fonctionnement automatique de l'éolienne et proposer une éolienne individuelle, de petite taille (3 à 6 mètres de diamètre) à la 
différence des anciens moulins à vent de taille plus importante (15 à 25 mètres de diamètre) comme le montre la série des timbres de 1981.

Halladay n'est ni le premier, ni le dernier à s'intéresser aux éoliennes, ni aux dispositifs de régulation de puissance : on recense aux Etats-Unis 56 brevets pour des moulins à vent (windmill, wind wheel) et 41 pour des régulateurs (governor) entre 1849 et 1877 . L'idée de faire « varier la surface motrice des ailes » selon les termes de Jules-Alexandre Granvoinnet [GRA 75] n'est pas nouvelle. L'ingénieur et physicien Jacob Leupold [LEU 25] décrit en 1725 dans son Théâtre des machines hydrauliques « une machine très simple dont les ailes ne peuvent être endommagées par la tempête »: les ailes peuvent pivoter autour de leur axe avec des ressorts de rappel. En 1745, le mécanicien anglais Edmund Lee dépose un brevet, modifié en 1772 par le meunier Andrew Meikle qui remplace le contrepoids par des ressorts (spring sail). Perfectionné par John Barber en 1772 et par Benjamin Heame en 1787, William Cubitt, fils de meunier, donne à cette idée sa forme aboutie en 1807 en utilisant des persiennes [MAY 75]. L'Angleterre, terre d'invention de ce dispositif, est aussi sa terre d'adoption. De nombreux moulins équipés d'ailes traditionnelles sont convertis à ce système, certains ne comportant que deux ailes à persiennes pour limiter le coût de la transformation.

\section{L'homme d'affaires John van Nortwick, directeur du Chicago, Burlington and Quincy Railroad} propose à John Burham de s'installer à Batavia, Illinois : la société United States Wind Engine and Pump Co. est créée en 1857. Un nouveau brevet est obtenu en 1877 avec un rotor dit sectionnel [HAL 77] : il ne s'agit plus de la machine brevetée en 1854, mais d'une machine dotée de huit sections comportant chacune dix ventelles (fans) solidaires et articulées autour d'un axe transversal : par grand vent, les différentes sections de l'éolienne se replient pour s'effacer au vent (visible sur l'image de gauche de la Figure 4).

United States Wind Engine and Pump Co. propose dans son catalogue de 1879 quatre types d'éoliennes : une éolienne de pompage pour l'alimentation d'abreuvoirs, de réservoirs d'eau pour les chemins de fer et la lutte contre les incendies en ville, une éolienne entraînant des machines agricoles (power windmill, "éolienne de force») et une (grande) éolienne de meunerie, qui n'utilise pas le brevet de 1877. L'éolienne de force non seulement fournit de l'eau, mais de l'énergie pour la laiterie y inclus le lavage des casseroles et des eaux, mais peut hacher la paille, battre et moudre, scier du bois et, ajoute le fabricant, deux de ses tâches peuvent être exécutés en même temps [MOT 80]. Le marché est encore hésitant en 1879 et, de cette gamme initialement large, seule l'éolienne de petite taille, à usage unique - le pompage - et individuel connaîtra un véritable succès.
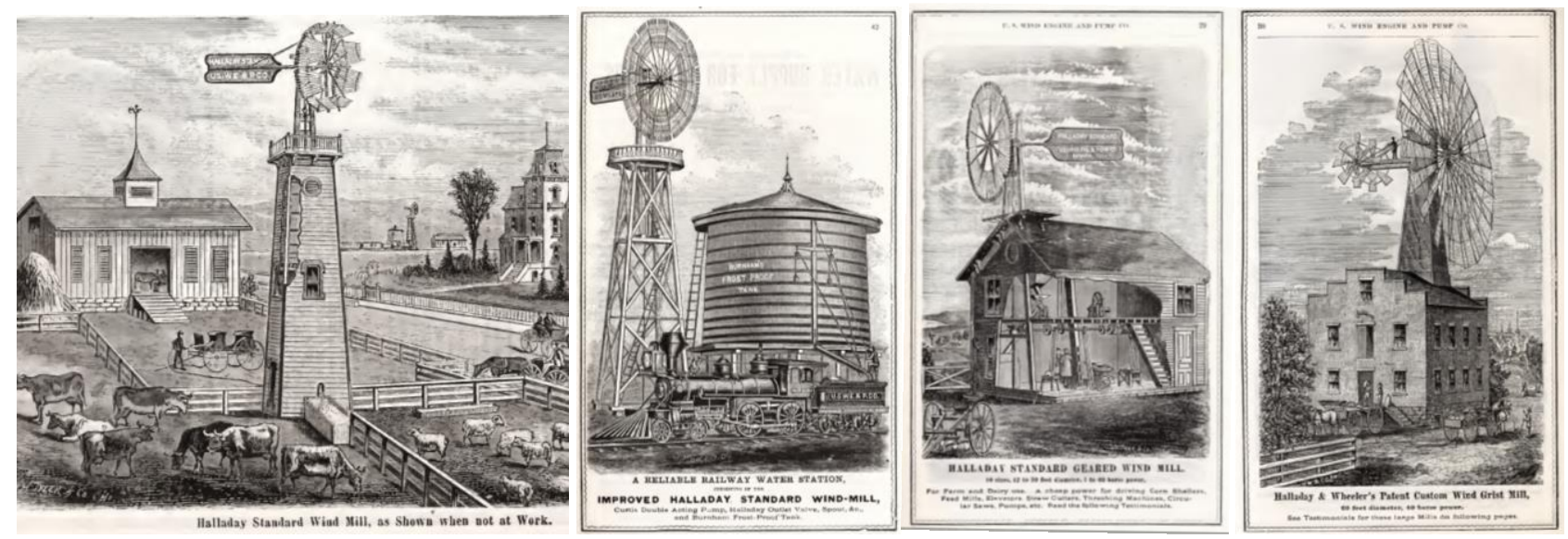

Figure 4. Trois marchés de l'éolienne américaine [USW 79]

La technique de vente est innovante et repose sur le chemin de fer : les vendeurs transportent une éolienne de démonstration par train, l'installent à la dernière gare et envoient les commandes par télégraphe. La machine est alors expédiée par train et assemblée sur site. L'éolienne de pompage suit le 
train, qu'elle alimente en eau et le train progresse avec la «Frontière » à une vitesse foudroyante : entre 1880 et 1890, la longueur des voies ferrées aux Etats-Unis croit de 88\% (120 $000 \mathrm{~km}$ construits), une croissance qui atteint $222 \%$ au Texas et $160 \%$ au Kansas [ADA 90].

Entre temps, le Révérend Leonard Wheeler invente en 1867 un nouveau système d'orientation avec palette latérale entraînant l'ensemble du rotor hors du vent. Cette dernière solution sera utilisée par tous les constructeurs, y compris US Wind Engine (Model E) au début du XXème siècle. Les fabricants sont très nombreux (77 fabricants en 1890), mais leur différentiation est faible. Les principales innovations sont la substitution du métal au bois pour les pales, la lubrification automatique et la démultiplication arrière (back geared) permettant au rotor de démarrer dans des vents plus faibles. La machine devient plus complexe, plus chère, mais aussi automatique et sans entretien, avec pour effet paradoxal de favoriser l'absence de maintenance entraînant une destruction prématurée de la machine, comme le regrette Paul Fuller en 1910 [FUL 10].

\subsection{Eolienne de pompage, machine de pionnier?}

En 1914, une éolienne Halladay de 12 pieds sur un pylône de 60 pieds coûte 132 dollars, sans compter les 169 dollars de forage de 100 pieds en 4 pouces de diamètre [SCH 14]. Cette somme est importante quand on la compare à la valeur moyenne de l'outillage et des machines agricoles sur une ferme américaine en 1910, d'environ 250 dollars dans l'Ouest américain, suivant les statistiques de 1919 [VAL 20]. La diffusion de l'éolienne américaine repose certes sur le pionnier individuel, mais sur un pionnier à tout le moins aisé, et surtout sur des spéculateurs fonciers (land speculator) qui disposent eux de moyens importants et prennent la main sur plus de la moitié des terres [HOL 76]. Le ranch texan XIT d'une surface de $12000 \mathrm{~km}^{2}$ entouré d'une clôture de $10000 \mathrm{~km}$ possède 335 éoliennes en 1900 [AND 10] et le King Ranch installe 265 éoliennes de pompage entretenues par cinq équipes de trois mécaniciens (windmillers) [ORU 40]. La statistique comme souvent masque des disparités très importantes : comment comparer le pionnier disposant de cent-soixante acres (65 hectares dans le cadre du Homestead de 1862) avec l'entreprise propriétaire de trois millions d'acres, près de 20000 fois plus?

Alimentant les abreuvoirs de l'éleveur, l'irrigation des champs ou l'arrosage des jardins, l'éolienne de pompage est aussi une machine qui permet la survie lors de période de sécheresse. Comme le constate le géologue Willard Johnson en 1900, lors de la sécheresse de 1893-1896, l'éleveur qui n'a pas voulu fuir ne fait que «tenir » ("holding on") au pied de son éolienne dans l'attente d'un changement de climat [JOH 01], une attitude hérö̈que. Mais, ajoute Johnson, ce pionnier ne pense qu'à cultiver de nouveau des céréales destinées à l'exportation : il ne cherche pas à adapter sa culture au climat semi-aride, mais à forcer la terre à accepter les céréales qu'il souhaite exporter. La force de ce mythe d'une «conquête technologique » de l'Ouest contribuera au désastre du Dust Bowl des années 1930 quand les fermiers persisteront à développer des cultures traditionnelles dans un milieu de plus en plus aride au lieu d'adapter ses cultures au milieu [CRO 92].

\subsection{Eolienne icone de l'américanisation}

L'historien Walter Prescott Webb propose dans son ouvrage The Great Plains [WEB 31] de considérer le barbelé, le six-coups et l'éolienne de pompage comme les trois techniques ayant permis la conquête de l'Ouest, la nouvelle Frontière. L'équipement technique du pionnier est simple car ce dernier est ingénieux, acharné, individualiste et n'a que peu de besoins. Cet esprit pionnier reste un mythe fort aux Etats-Unis, une forme de nostalgie au pays de l'abondance et ce mythe agit encore de nos jours : du barbelé au mur avec le Mexique, du six-coups au port d'arme généralisé, de l'éolienne autonome au gaz de schiste, les mêmes ressorts sont à l'œuvre pour structurer l'american way of life.

L'éolienne de pompage est aussi un phénomène industriel, fabriquée à plusieurs millions d'exemplaires, exportée dans le monde entier. La grande différence entre l'éolienne Halladay et tous ses supposés prédécesseurs européens est l'industrialisation dont elle fait l'objet, sur un mode proche 
de ce qui sera fait avec l'automobile comme le remarque Webb. C'est l'american system of manufacturing se fondant sur la mécanisation de la production et l'interchangeabilité des composants. La construction est modulaire, l'éolienne est le plus souvent livrée en kit, transportée en caisse de bois, pouvant être montée et démontée avec des outils agricoles classique. De ce point de vue, l'éolienne d'Amédée Durand en France (une cinquantaine de machines fabriquées de 1828 à 1860) n'a que peu à voir avec l'éolienne américaine, même si René Champly estime en 1933 que «cette invention fut reprise par les Américains» [CHA 33], ou que plus récemment Etienne Rogier affirme en 2014 qu'Amédée Durand «a tout de même précédé d'un bon quart de siècle l'Américain Halladay » [ROG 14]. Là encore, tout est question de définition.

\section{L'éolienne danoise}

\subsection{Un siècle d'invention de l'éolienne moderne}

L'histoire de l'invention de l'éolienne électrique contemporaine, l'« éolienne moderne », est résumée en quatre dates par une série de timbres émis par la poste danoise en 2007 : d'abord les recherches de Poul La Cour au tournant du XXe siècle (Askov dans le Jutland, 1891), ensuite le relai d'un de ses élèves Johannes Juul (Gedser sur l'île de Sjælland, 1957) et enfin la redécouverte par des mécaniciens ruraux dans les années 1970 donnant naissance à l'industrie éolienne danoise avec des éoliennes «terrestres » du fabricant danois Vestas (île de Bogø, 1989) et des éoliennes «en mer»du fabricant lui aussi danois Bonus (Middelgrunden au large de Copenhague, 2000).

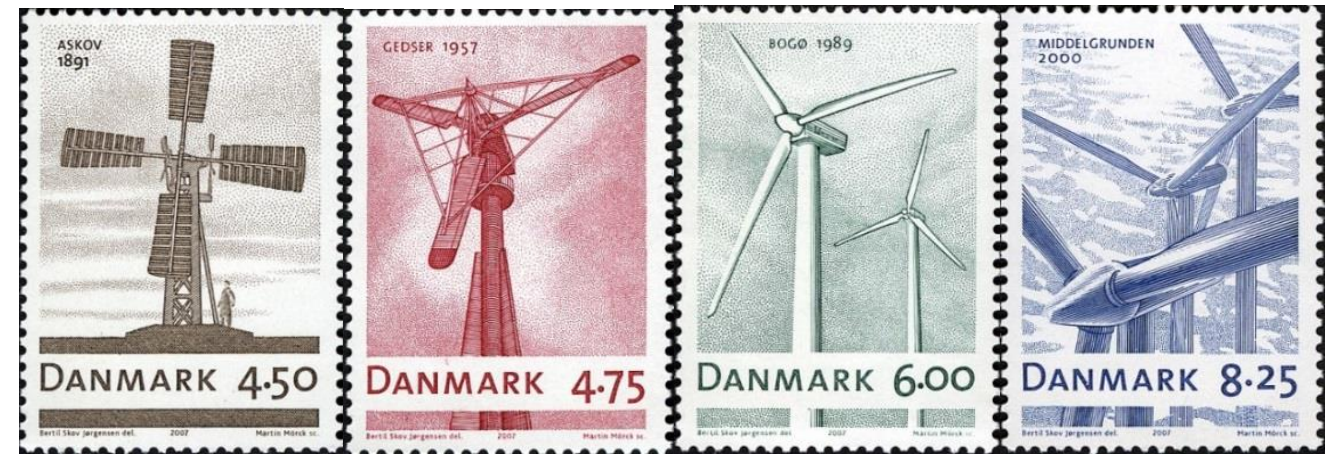

Figure 5. Série des quatre timbres sur les éoliennes danoises, édités par le poste danoise en 2007 [POS 07].

Poul La Cour, physicien et pédagogue, s'installe dans le Jutland (Ouest du Danemark) pour enseigner dans l'école d'Askov. L'éolienne La Cour est un moulin doté de quatre pales et d'un système de persiennes s'ouvrant quand le vent est trop élevé (voir Cubbitt évoqué plus haut), une machine fournie par l'entreprise N.J. Poulsen d'Esbjerg. Le remplacement des voiles par des profils minces, une tôle pliée, permet d'obtenir de meilleures performances, sans pour autant dépasser celles obtenues par l'ingénieur anglais John Smeaton en 1760, ni d'ailleurs celles des éoliennes américaines de pompage.

Une société d'ingénierie éolienne est créée et, si les apports en terme d'aérodynamique sont faibles, c'est dans le domaine de l'ingénierie éolienne que Poul La Cour innove avec la création de la société DVES (Dansk Vind Elektrisitets Selskab, Société Danoise d'Electricité Eolienne) qui propose un modèle original de diffusion de centrales électriques dans le milieu rural danois. Des dizaines de centrales éoliennes rurales vont être installées alimentant de grosses fermes, des industries agroalimentaires et des municipalités. La mort de La Cour en 1909 ralentit le développement et la société DVES cesse ses activités en 1919.

C'est un ancien élève de La Cour qui redéveloppe le moulin électrique dans le cadre de la compagnie d'électricité SEAS (Sydøstsjallands Elektricitets Aktieselskab). Johannes Juul, électricien et inventeur, teste différents rotors en soufflerie et développe trois prototypes de machines de production d'électricité raccordées au réseau. Installée à Gedser en 1957, la dernière machine 
fonctionne jusqu'en 1967 et fût abandonnée après la mort de Juul en 1966. Il n'existe pas de données opérationnelles du fonctionnement de cette machine, mais son surnom de oil mill, «moulin à huile » (les projections d'huile sont fréquentes) indique un fonctionnement erratique. Dix ans plus tard, les ingénieurs de la NASA, dans le cadre de leur programme de développement éolien, subventionnent des essais sur cette machine abandonnée depuis dix ans [LUN 80]. Rénovée au début des années 1990, la nacelle de cette machine est présentée au Musée de l'Energie du Danemark.

Au milieu des années 1970, différents mécaniciens ruraux développent des machines éoliennes de production de chaleur et d'électricité en réponse à la «crise de l'énergie » qui a lourdement touché le Danemark en 1973. De multiples petits constructeurs apparaissent, utilisant des solutions techniques variées. A Tvind, des professeurs d'une école technique installent en 1978 une grande éolienne de 2 mégawatts qui constitue une première. Le paysage technique est cependant dominé des entrepreneurs individuels, qui se définissent eux-mêmes, selon les termes d'Erik Grove-Nielsen, fondateur du fabricant de pales Okaer Energi, comme « individualistes travaillant avec d'autres individualistes dans le pays entier » [GRO 18]. Il choisit même de faire tourner les pales qu'il fabrique dans le sens opposé à celles de Tvind, jugée trop «collectiviste », alors qu'il utilise le moule du premier prototype PTG de $15 \mathrm{~kW}$ de l'équipe de Tvind.

L'éolienne danoise se caractérise par un rotor tripale orienté face au vent, régulation par décrochage aérodynamique et génératrice asynchrone directement raccordée au réseau. Cette machine intègre dans une même conception des solutions techniques éprouvées sur d'autres éoliennes danoises : l'éolienne Agricco à génératrice asynchrone et calage variable raccordée au réseau en 1919, une première mondiale, les éoliennes $F$. S. Smidth bipale avec frein aérodynamique dans les années 1940. L'éolienne de Gedser est un prototype certes innovant, mais sans développement industriel, sauvé de la destruction par les américains : la filiation entre Poul La Cour et l'éolienne moderne est de ce point de vue fragile.

Toutes ces innovations, issues de savoir-faire de mécaniciens ruraux et de prototypes, n'aurait pas débouché dans la présence d'industriels comme Vestas. Les racines de Vestas remontent au tournant du XXe siècle quand H. S. Hansen installe en 1898 un atelier de mécanique près de la gare de Lem [WUS 12]. En 1945, son fils crée Vestjysk Staalvarefabrik (Fabrication de produits en acier du Jutland de l'Ouest, d'où le nom de Vestas) spécialisée dans le matériel pour l'agriculture et l'industrie agroalimentaire. Une nouvelle diversification est effectuée en 1960 avec la fabrication de grues hydrauliques pour camions. La «crise de l'énergie » touchant de plein fouet le secteur des transports, Vestas se lance dans les éoliennes avec un prototype d'éolienne à axe vertical monté en 1978. Cette expérience est un échec et Vestas se tourne vers une petite entreprise de mécanique, HVK (Herborg Vindkraft), animée par un ingénieur et un mécanicien, et lui achète une licence en 1979. Les éoliennes Vestas HVK 10 et Vestas HVK 15 sont les premières à être fabriquées, l'argumentaire commercial insistant sur la sécurité de fonctionnement de ces machines (triple sécurité avec frein à disque sur l'arbre principal, décrochage aérodynamique et frein aérodynamique à l'extrémité des pales se déployant automatiquement en cas de survitesse). Vestas bénéficie aussi des avis du Centre d'essais d'éoliennes créé en 1978 au sein du Centre de recherche nucléaire Risoe, l'homologation de l'éolienne permettant au client de bénéficier d'une subvention de $30 \%$ du coût de la machine.

Au début des années 1980, Vestas profite de l'essor du marché californien où il exporte des milliers de machines et bénéficie d'un retour d'expérience d'une valeur inestimable. C'est le début du développement de la société qui passe de 180 employés en 1980 à 800 en 1985 avec une usine de $12000 \mathrm{~m}^{2}$. En octobre 1986, c'est la faillitte avec une dette de 13 millions d'Euros. La famille Hansen est débarquée et la société est relancée sous un nouveau nom, Vestas Wind Systems A/S, dirigée par Poulsen avec 60 employés. Le succès est foudroyant et en 1998, la société entre en bourse et récolte 175 millions d'Euros, le capital ayant été souscrit huit fois. 


\subsection{Eolienne, canon de la « façon de faire » danoise}

En 2006, le ministre de la Culture Brian Mikkelsen, du Parti populaire conservateur (Det Konservative Folkeparti), publie les «canons culturels», destinée à être une introduction au patrimoine culturel danois. L'éolienne de Gedser est l'un de ces canons culturels considérant que ce travail de pionnier a joué un rôle important dans la position actuelle de l'industrie éolienne danoise et que « cette industrie fait partie du concept de Danish Design comme B\&O (Bang \& Olufsen) ou la lampe $\mathrm{PH}$ », constituant « une caractéristique d'une partie du paysage danois » (en karakteristisk del af det danske landskab) [KUL 07].

En lien avec la société d'histoire de l'énergie éolienne (Danmarks Vindkrafthistoriske Samling, DVS), le musée de l'énergie (Energimuseet) et le centre nordique d'information sur les énergies renouvelables (Nordisk Folkecenter for Vedvarende Energi), une histoire de l'éolien au Danemark se construit, ancrant le fort engagement actuel du pays dans l'énergie éolienne dans plus de cent ans de travaux de pionniers visionnaires, symbolisant la «manière de faire danoise » (the Danish Way) à la base du «modèle énergétique danois» (The Danish Energy Model) [WIN 09]. Ce récit historique présenté plus haut est repris par les industriels danois comme le fabricant de pales d'éolienne LM Wind Power, employant 5000 personnes dans le monde, dont la storytelling raconte une histoire de l'éolien en cinq images : le Drakkar viking, le moulin traditionnel, le moulin de La Cour (1891), le moulin Agricco (1919) et les éoliennes modernes [LMW 18].

Ce discours sur ces (petits) mécaniciens ruraux damant le pion aux (grandes) entreprises allemandes (Growian de M.A.N.) ou américaines (série des MOD-X de la NASA) au début des années 1980 reçoit un accueil favorable dans le monde de la sociologie de l'innovation au point de masquer l'identité de l'éolienne danoise, sa définition même : une machine industrielle, sûre et performante, fabriquée en série et n'utilisant que des techniques éprouvées. Quand, au milieu des années 1990, la demande politique, européenne et nationale, exigera des machines de plus grande taille - les éoliennes actuelles la réticence est grande dans le milieu industriel danois et la main est reprise par de nouvelles entreprises allemandes qui n'hésiteront pas à utiliser des techniques testées par les (grandes) entreprises du début des années 1980 (génératrice synchrone à vitesse variable, convertisseur électronique de puissance, calage de pale). Là encore, la filiation entre l'éolienne danoise des années 1980 à 1995 et les éoliennes « modernes » n’est pas aussi directe.

\section{Question de définition}

20000 modèles ! s'exclame le technologue Yves Deforge au sujet du nombre de bougies que revendique avoir développé un constructeur allemand en 1977 pour le moteur à combustion interne. Il ajoute «puisque c'est « l'échelle qui crée le phénomène (ou qui l'estompe) il s'agit bien de choisir le « pouvoir séparateur » de l'instrument d'observation. Entre une lignée fondée sur un principe unique et 20000 descriptions détaillées prenant en compte toutes les particularités des technologies et des formes, la plage d'ajustement est étendue. » [DEF 85] Le même constat pourrait être fait sur les éoliennes évoqués dans les timbres: entre les quatorze types, 31 fonctions et 34 caractéristiques des molinologues hollandais (soit 14576 combinaisons qui ne sont heureusement pas toutes réalisées, mais il ne s'agit que des moulins des Pays-Bas...) et le « moulin hollandais » du graphiste, comment définir ce dont on parle ? D’autant que ce « moulin hollandais » tire son origine des Flandres...

\subsection{De la machine au monument}

Les trois récits philatéliques et historiques qui viennent d'être analysés parlent tous d'une machine dans son sens le plus général, un outil perfectionné qui «supplée au nombre ou à l'adresse des ouvriers » selon Jean Hachette [HAC 19]. Il s'agit toujours d'une machine composite avec une première machine, un moteur, qui a pour objet «l'emploi et l'économie des forces motrices » et une seconde 
machine, un récepteur, qui «modifie l'action de ce moteur suivant le but que l'on se propose » (drainer, pomper, produire de l'électricité).

Dans ce cadre d'analyse, une éolienne américaine de pompage est une éolienne (moteur primaire) qui entraîne une pompe (moteur secondaire) pour produire un effet utile, élever de l'eau. L'histoire de cette éolienne de pompage est le croisement d'une histoire d'éoliennes et d'une histoire de pompes. Une éolienne de pompage (pumping windmill pour le fabricant d'éoliennes [SEA 03]), une éolienne entraînant une pompe, est aussi une pompe éolienne (wind mill pump pour le fabricant de pompes [GOU 00]), une pompe entraînée par une éolienne. Dans le premier cas, il s'agit d'une « source d'énergie » en langage contemporain, dans le second cas d'une production utile pour l'utilisateur. De tout cela, moteur transmission et récepteur, l'utilisateur final n'en a cure : la machine est un tout, elle utilise le vent et alimente en eau son jardin ou son abreuvoir, c'est un moyen de pompage autonome. Il a le choix entre une pompe à main, une pompe à moteur thermique ou une pompe électrique : pomper est un service de base, le choix de l'énergie étant second.

Une seconde remarque d'Hachette attire l'attention : « il n'y a qu'une seule espèce de Machine qui reçoive directement l'action du vent, pour la transmettre à l'arbre de rotation d'un moulin, qui est horizontal ou vertical, selon la forme que l'on donne aux ailes » à la différence ajoute-t-il des Machines qui reçoivent directement l'action de l'eau : (roues hydrauliques par-dessus et en-dessous, bélier hydraulique). En résumé, pour le technologue, toutes les éoliennes sont munies d'ailes et, en allant plus loin, toutes les éoliennes ayant connu une large diffusion sont munies d'ailes utilisant le phénomène physique de portance aérodynamique : voilà la "lignée fondée sur un principe unique » dont parle Yves Deforge et une définition élargie de l'éolienne. Ce principe unique connaît des variations géographiques au gré des transferts, des migrations humaines, des analogies avec d'autres techniques utilisées localement. En certains endroits, il y a résonance entre une somme d'innovations potentielles et un usage particulier qui donne une forme à l'objet technique « éolienne ». Comme le dit Fernand Braudel [BRA 92], « il y a toujours présentes, des centaines d'innovations possibles, endormies en somme et qu'il devient urgent, un beau jour, de réveiller. » Le molinologue s'attache à recenser ces centaines d'innovations, les différences de forme et de structure, les emprunts et remaniements, les adaptations de mécanismes pour un usage particulier avec des inventeurs, ces héros qui s'acharnent à mettre au point une machine nouvelle. L'éolienne s'ancre dans un territoire, dans une histoire et devient monument, objet de musée, et parfois icône nationale et timbre.

\subsection{De la source d'énergie renouvelable à l'atténuateur climatique}

Dans son analyse des énergies aux Pays-Bas au XVIIe siècle, Jan de Zeeuw estime à 45 milliards de kilocalories la production des 3000 moulins à vent de l'époque, une production insignifiante $(0,75 \%)$ par rapport à celle de la tourbe évaluée à 6000 milliards de kcal [ZEE 78]. Comment peut-on mettre sur un même plan des moulins à vent et de la tourbe, ou aujourd'hui des éoliennes modernes et du charbon ou du nucléaire ? C'est là le mystère du concept d'énergie, une abstraction confondue longtemps avec les concepts tout aussi abstraits de «force» ou de «travail»: il suffit de consulter les articles correspondants dans le Grand Dictionnaire Universel du XIXe siècle [LAR 66]. Définir une éolienne comme source d'énergie renouvelable, c'est se focaliser sur le moteur primaire en le séparant du récepteur secondaire qui lui donne pourtant sa raison d'être (pomper, moudre, broyer...). Mais, à regarder de plus près, l'éolienne est devenue aujourd'hui une source d'énergie électrique renouvelable : elle produit un medium invisible qui vient irriguer l'ensemble des économies modernes en s'adaptant à tous les usages, en rendant tous les services à condition d'être raccordé au réseau transnational.

Dans notre monde électrique, atténuer les effets du changement climatique passe par la production d'électricité peu émettrice de gaz à effet de serre, mettant l'éolienne au cœur des stratégies climatiques. Si l'éolienne a difficilement trouvé sa place sur le réseau électrique dans les années 1990, la situation 
se retourne aujourd'hui, les industriels de l'éolien réclamant l'extension de l'électrification, notamment pour le chauffage et les transports [WIN 18].

\section{Conclusion}

Entre une éolienne unique dans une définition floue en grand angle ou des éoliennes multiples dans une définition précise et focalisée, quel «pouvoir séparateur» adopter? Existe-t-il un «niveau judicieux d'analyse », un «niveau moyen d'abstraction » pour reprendre les termes d'Yves Deforge ? De tout ce qui précède, les deux niveaux de définition doivent être adoptés simultanément, analyser les innovations locales à l'aulne de l'innovation globale. Dans cette vue, l'innovation il n'existe d'une seule « éolienne » qui remonte à la fin du XIIème siècle avec le moulin pivot prend différentes formes dans différents contextes d'usage précis, situés dans le temps et dans l'espace, en mobilisant les technologies disponibles à l'épouqe.

Ce que nous montrent aussi ces exemples de timbres nationaux, c'est le fait que chaque machine vient résoudre un problème posé par l'usage d'autres techniques. Le moulin de drainage s'installe sur un polder créé par l'exploitation humaine de la tourbe. L'éolienne de pompage se diffuse pour imposer des cultures céréalières en milieu semi-aride. L'éolienne danoise émerge dans un pays soumis à embargo au cœur des crises pétrolières des années 1970. L'innovation technique est un moyen d'éviter la difficile question du pourquoi de ces «crises». Entre remise en question et avancée héroïque, l'homme choisit son camp : on ne renonce pas, on progresse, l'innovation est un combat : Lion Batave contre loup d'eau, pionnier américain contre milieu semi-aride, mécanicien danois contre effondrement énergétique de la civilisation occidentale... Le choix le plus sûr est d'innover, la technique est reine, comme l'écrivait Fernand Braudel écrivait en 1979 [BRA 92] :

«[...] aujourd'hui une grande crise générale met toutes économies développées au pied du mur : innove, ou meurs, ou stagne ! Elles choisiront sûrement d'innover. Pareille mise en demeure a précédé sans doute, chacune des grandes relances de la croissance économique qui, depuis des siècles et des siècles, ont toujours eu un support technique. En ce sens, la technique est reine : c'est elle qui change le monde. »

Question de définition, question d'échelle, question de focale, en somme.

\section{Références}

[ADA 90] ADAMS Henry C., Report on transportation business in the United at the Eleventh Census: 1890, Part I Transportation par Land, Washington DC, Government Printing Office, 1895, p. 4-5.

[AND 10] ANDERSON H. Allen, "XIT RANCH," Handbook of Texas Online, Texas State Historical Association, 2010. http://www.tshaonline.org/handbook/online/articles/apx01.

[APP 06] Appleton Manufacturing Co., Illustrated Catalogue of Farm Machinery, Batavia, Appleton Mfg Co, 1906.

[ARR 18] «Arrêté du 17 juillet 2018 complétant le programme philatélique de l'année 2019 et fixant le programme de l'année 2020 », M. le Ministre de l’Economie et des Finances, Journal Officiel de la République Française, nº165, 20 juillet 2018.

[BAK 80] BAKER T. Lindsay, «Turbine-Type Windmills of the Great Plains and Midwest », Agricultural History, vol. 54, $\mathrm{n}^{\circ} 1$, p. 3851, 1980.

[BRA 92] BRAUDEL Fernand, Civilisation matérielle, économie et capitalisme, XVe-XVIIIe siècle. 1 - Les Structures du quotidien, Paris, Armand Colin, 1992 [1979], p. 382.

[CHA 33] ChAMPLY René, Les Moteurs à vent. Théorie, construction, montage, utilisation au puisage de l'eau et à la production de l'électricité, Paris, Dunod éditeur, 1933, p. 2.

[CIR 91] CIRIACono Salvatore, "Venise et la Hollande, pays de l'eau (XVe-XVIIIe siècle)», Revue historique, CCLXXXV/2, p. 295-320, 1991. 
[CRO 92] CRONON William, «A Place for Stories: Nature, History, and Narrative », The Journal of American History, vol. 78, no 4, p. 13471376, 1992.

[DAM 01] DAM Petra J. E. M. van, « Sinking Peat Bogs: Environmental Change Holland, 1350-1550», Environmental History, vol. 6, $\mathrm{n}^{\circ}$ 1, p. 3245, 2001.

[DEF 85] Deforge Yves, Technologie et génétique de l'objet industriel, Paris, Maloine, 1985, p. 102.

[DEH 01] De Hollandsche Molen, De Molendatabase - Allemolens in Nerderland en België, 2001-2018. « Ondermolen D (Museummolen » https://www.molendatabase.nl/nederland/molen.php?nummer=713

[DEN 06] DenEwetT Lucien, « Niet Nederland maar vlaanderen de bakermat! Belangrijke archiefvondst. De eerste vermelding van een poldermolen : de nog bestaande 'hoesse molen' bij Gent (1316) », Molenecho's. Vlaams tijdschrift voor Molinologie, vol. 34, no 3, p. 170190, 2006.

[FUL 10] FULLER Paul Edwin, «The Use of Windmills in Irrigation in the Semiarid West », Farmer's Bulletin 394 - U.S. Department of Agriculture, p. 144, p. 35-36, 3 mai 1910.

[GAT 42] Gates Paul Wallace, «The Role of the Land Speculator in Western Development », The Pennsylvania Magazine of History and Biography, vol. 66, n 3, p. 314-333, 1942.

[GOE 10] GOELDNER-GIANELLA Lydie, « Changement climatique et dépoldérisation : le rôle des acteurs et le poids des représentations sociales sur les côtes d'Europe atlantique », Quaderni. Communication, technologies, pouvoir, $\mathrm{n}^{\circ} 71$, p. $4160,2010$.

[GOU 00] The Goulds Manufacturing Company, Catalogue "A" of Pumps and Hydraulic Machinery for every service, 28th ed., Seneca Falls, NY, The Goulds Manufacturing Co., 1900, p. 40.

[GRA 75] GRANDVOInNET Jules-Alexandre, Le génie rural, Paris, Librairie scientifique, industrielle et agricole E. Lacroix, 1875, p. 160-166.

[GRO 18] GROVE-NIELSEN Erik, «Økær Vind Energi 1977 - $1981 »$, Winds Of Change : Stories of a dawning Wind Power Industry, http://www.windsofchange.dk/WOC-77-81.php

[HAC 19] HAChETTE Jean Nicolas Pierre, Traité élémentaire des machines, Paris, veuve Courcier, 1819, p. XIX.

[HAL 54] HALLADAY Daniel, « Wind Wheel - Improved Governor for Windmill », US Patent Office, Nr 11,629, Aug. 29 1854.

[HAL 77] HALLADAY Daniel, «Improvement in Windmills », US Patent Office, Nr 190,430, May 81877.

[HIL 96] HILLS Richard Leslie, Power from Wind: A History of Windmill Technology, Cambridge, Cambridge University Press, 1996, p. 119.

[HOL 76] HoltgRIEve Donald G., «Land Speculation And Other Processes In American Historical Geography », Journal of Geography, Vol. 75, No. 1, p. 53-64, 1976.

[JOH 01] Johnson Willard D., « The High Plains and Their Utilization », in WALCOTT Charles D. (dir.), Twenty-First Annual report of the Director of the United States Geological Survey, 1899-1900: Part IV - Hydrology, Washington, Government Printing Office, 1901, p. 601-741, p. 690.

[JOR 63] JORDAN Terry G., «Windmills in Texas », Agricultural History, vol. 37, n 2, p. 8085, p. 83, 1963.

[KUL 07] Kulturkanon i undervisnoingen - Inspiration og forslag, http://www.kulturkanon.emu.dk/

[LAR 66] Larousse Pierre (dir.), Grand Dictionnaire Universel du XIXe siècle, Paris, Larousse, 1866-1876.

[LEU 25] LEUPOLD Jacob, Theatrum machinarum hydraulicarum oder, Schau-Platz der Wasser-Künste, Tomus II, Leipzig, 1725, §128 et Tab XXXIII.

[LMW 18] LM WIND POwER, « History of wind », 2018, http://www.lmwindpower.com/Wind-Technology/The-case-forwind/ History-of-wind

[LUN 80] LundSAGer P., Fransen S. et Christensen Clayton M., Analyis of Data from the Gedser Wind Turbine 1977-1979, RISO-M-2242, Roskilde, Denmark, Risoe National Laboratory, 1980.

[MAE 13] MAegaARd Preben, Krenz Anna et Palz Wolfgang (eds.), Wind Power for the World - The Rise of Modern Wind Energy, s.1., Taylor \& Francis Group, LLC (coll. « Pan Stanford Series on Renewable Energy »), 2013.

[MAY 75] MAYR Otto, The Origins of Feedback Control, Cambridge, The MIT Press, 1970, p. 90-108.

[MOT 80] « Motive Powers for Farms », Cultivator \& Country Gentleman, vol. 45, n 1412, p. 119, 19 Feb. 1880. 
[MUL 91] MULDER Karel F., «A Useful Tool Turned into a Monument: Controversies over Holland's Windmills in the First Half of the 20th Century », IA. The Journal of the Society for Industrial Archeology, vol. 17, no 2, p. 3746, 1991.

[MUS 18] MUSEUMMOLEN, Noordervaart 2, 1636 VL Schermerhorn. https://www.museummolen.n1/ ; Museumgemaal Wilhelmina, Molendijk 9, 1636 VK, Schermerhorn. https://www.museum gemaalwilhelmina.nl/

[NET 13] NeTHERLANDS ENVIRONMENTAL ASSESSMENT AGENCY, The effects of the climate change in the Netherlands: 2012, The Hague, Planbureau voor de Leefomgeving (PBL), 2013.

[NIE 12] Nieuwstadt Mark van, Minotaurus et MAARn, A future for mills - Principles for dealing with heritage mills, Amersfoort, Cultural Heritage Agency, 2012. https://cultureelerfgoed.nl/dossiers/molens/

[ORU 40] ORUM A., «The King Ranch », Iowa State University Veterinarian, 1940, vol. 2, no 2. Résumé de l'article « The World's Biggest Ranch », Fortune, p. 49, December 1933.

[PET 13] Peterson Stacey L. et Scheetz George H., Windmill City : A Guide to the Historic Windmills of Batavia, Illinois, Batavia Public Library, 2013 [2008].

[POS 07] Post DENMARK, «Danish Windmills : Askov 1891 [DK005.07], Gedser 1957 [DK006.07], Bog $\varnothing 1989$ [DK007.07], Middelgrunden 2000 [DK008.07] », January 10, 2007.

[POS 13] POSTNL, « Dutch windmills on stamps », Press Release, The Hague, 13 June 2013.

[POS 14] POSTNL, « Dutch icons feature on new basic stamp range », Press Release, The Hague, 3 January 2014.

[ROG 14] RoGIER Etienne, «Le Moteur à Vent de Roger Amédée », Systèmes Solaires, no 222, p. 5861, 2014.

[SEA 03] SEARS, RoEBUCK \& Co, A catalogue of windmills and supplies, Chicago, Ill., Sears, Roebuck \& Co., 1903, p. 12.

[SCH 14] SchroEDER Glenn Wilson, Systems of water supply for a 320-acre dairy farm, Bachelor of Science in Agronomy, University of Illinois, Urbana-Champaign, 1914.

[STA 20] StATISTICAL AbSTRACT OF THE United StATES: 1919, Washington D.C., Bureau of the Census Library, 1920.

[STE 30] STERCK J.F.M. et all., De werken van Vondel. Deel 4. 1640-1645, Amsterdam, 1930, p. 296.

[TEB 02] TEBRAKE William H., «Taming the Waterwolf: Hydraulic Engineering and Water Management in the Netherlands during the Middle Ages », Technology and Culture, vol. 43, n 3, p. 475-499, 2002.

[USP 10] United States Postal Service, Stamp Announcement 11-04: Kansas Statehood, USPS Government Relations and Public Policy, 30 Dec. 2010.

[USP 81] United States Postal SeRvice, Windmills, Scott Catalogue USA: \#1738-\#1742, 7 Feb. 1981 ; Flags of Our Nation - Illinois, WNS US108.08, 2 Sept. 2008 ; Kansas Statehood, WNS US002.11, 27 Jan. 2011, Topeka KS.

[USW 79] U.S. Wind ENGINE \& PUMP Co., Descriptive catalogue, 12th ed., Batavia, Illinois, U.S. Wind Engine \& Pump Co., 1879 , p. 11.

[VAL 20] «Value of Farm Property: By States and by Geographical Divisions, 1890, 1900, and $1910 »$, Statistical Abstract of the United States 1919, Washington, Bureau of the Census Library, 1920, p. 141-144.

[VES 07] VESTAS WIND SYSTEMS A/S, Discover the unique power of the wind, Print version of www.vestas.com/dupowDK/, 2007.

[WEB 59] WeBB Walter Prescott, The Great Plains - A study of institutions and environment, Waltham, Mass., Blaisdell Pub. Co, 1959 [1931], p. 340.

[WIN 09] Wind Power - The Danish Way - From Poul la Cour to Modern Wind Turbines, Askov, Danemark, The Poul la Cour Foundation, 2009 ; DANISH ENERGY AGENCY, The Danish Energy Model - Innovative, Efficient and Sustainable, Copenhagen, Danish Energy Agency, 2015.

[WIN 18] WINDEUROPE, Electrification the key to decarbonising Europe's economy, Press Release, 10 July 2018. https://windeurope.org/newsroom/press-releases/electrification-the-key-to-decarbonising-europes-economy/

[WUS 12] WÜSTENHAGEN Rolf, « Growing with the Wind: The Case of Vestas » dans Diversity in European Marketing, Wiesbaden, Germany, Gabler Verlag, 2012, p. 253272.

[ZEE 78] ZEEUw Jan W. de, «Peat and the Dutch Golden Age. The Historical Meaning of energy-attainability », A.A.G. Bijdragen (Landbouwuniversiteit Wageningen), vol. 21, p. 3-31, 1978. 